\title{
CORRIGENDUM
}

\section{The protective effect of the obesity-associated rs9939609 A variant in fat mass- and obesity-associated gene on depression}

Z Samaan, S Anand, X Zhang, D Desai, M Rivera, G Pare, L Thabane, C Xie, H Gerstein, JC Engert, I Craig, S Cohen-Woods, V Mohan, R Diaz, X Wang, L Liu, T Corre, M Preisig, Z Kutalik, S Bergmann, P Vollenweider, G Waeber, S Yusuf and D Meyre

Molecular Psychiatry (2013) 18, 1324; doi:10.1038/mp.2012.178; published online 5 March 2013

Correction to: Molecular Psychiatry advance online publication 20 November 2012; doi:10.1038/mp.2012.160

The published article contains several errors, as described below.

1. The second author should have been listed as SS Anand, not S Anand.

2. Several of the authors' affiliations/departments have changed. The correct affiliation information is shown below:

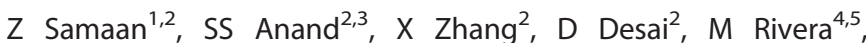

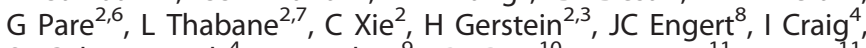

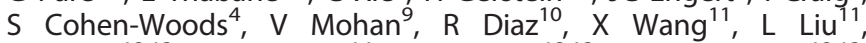
T Corre ${ }^{12,13}$, M Preisig ${ }^{14}$, Z Kutalik ${ }^{12,13}$, S Bergmann ${ }^{12,13}$, P Vollenweider $^{15}$, G Waeber ${ }^{15}$, S Yusuf ${ }^{2,3}$, D Meyre ${ }^{2,7}$

${ }^{1}$ Department of Psychiatry and Behavioural Neurosciences, McMaster University, Hamilton, ON, Canada;

${ }^{2}$ Population Health Research Institute, Hamilton, ON, Canada;

${ }^{3}$ Department of Medicine, McMaster University, Hamilton, ON Canada;

${ }^{4}$ MRC Social Genetic and Developmental Psychiatry Centre, London, UK;

${ }^{5}$ University of Granada, Granada, Spain;

${ }^{6}$ Department of Pathology and Molecular Medicine, McMaster University, Hamilton, ON, Canada;

${ }^{7}$ Department of Clinical Epidemiology and Biostatistics, McMaster University, Hamilton, ON, Canada;
${ }^{8}$ McGill University, Montreal, QC, Canada;

${ }^{9}$ Madras Diabetes Research Foundation, Chennai, India;

${ }^{10}$ ECLA—Academic Research Organization, Rosario, Argentina;

${ }^{11}$ Beijing Hypertension League Institute, Beijing, China; ${ }^{12}$ Department of Medical Genetics, University of Lausanne, Lausanne, Switzerland; ${ }^{13}$ Swiss Institute of Bioinformatics, Lausanne, Switzerland; ${ }^{14}$ Department of Psychiatry, CHUV, Lausanne, Switzerland; ${ }^{15}$ Division of Internal Medicine, CHUV, Lausanne, Switzerland

3. The information for the corresponding author was incomplete. The complete information is shown below:

Correspondence: D Meyre, Department of Clinical Epidemiology and Biostatistics, McMaster University, 1280 Main Street West, MDCL Room 3205, Hamilton, ON, Canada L8S 4K1.

E-mail: meyred@mcmaster.ca

4. The following statement was omitted from the Acknowledgements section:

SS Anand is supported by a Canada Research Chair in Ethnic Diversity and Cardiovascular Disease.

5. The authorship of ref. 31 was shown incorrectly. The correct reference is shown below:

Gerstein HC, Yusuf S, Holman R, Bosch J, Pogue J; DREAM Trial Investigators. Rationale, design and recruitment characteristics of a large, simple international trial of diabetes prevention: the DREAM trial. Diabetologia 2004; 47: 1519-1527. 